\title{
EL APORTE DE JUAN PABLO II A LA DOCTRINA SOCIAL DE LA IGLESIA*
}

\begin{tabular}{c}
\hline Luigi Negri** \\
Universidad Católica del Sacro Cuore \\
Obispado de San Marino-Montefeltro \\
segretario@luiginegri.it \\
\hline
\end{tabular}

ReSUMEN: El presente trabajo pretende evidenciar el aporte dejado por el papa Juan Pablo II para fortalecer la Doctrina Social de la Iglesia. En un primer momento, se analiza la herencia dejada por cuatro de sus antecesores, como fueron Pío IX, León XIII, Pío XI y Pío XII, quienes tuvieron una participación fundamental dentro de la vida de la Iglesia por afrontar los embates de la modernidad. En un segundo momento, nos detenemos en la figura de Juan Pablo II para realizar un análisis de su actuación en la vida de la Iglesia desde la perspectiva de la «nueva evangelización». Para tal efecto, se plantea una revisión de cuatro documentos en los que Juan Pablo II aborda aspectos fundamentales de la vida del hombre, como son Redemptor Hominis, Lavorem Excersens, Sollicitudo Rei Socialis y Centesimus Annus.

* Traducción de Ma. Teresa Briozzo Pereyra.

** Mons. Luigi Negri es doctor en Filosofía por la Universidad Católica del Sacro Cuore de Milán y licenciado en Teología. En el año 2005 fue ordenado Obispo de San MarinoMontefeltro. Es un gran estudioso del Magisterio Pontificio y de la Doctrina Social de la Iglesia. Ha escrito más de 30 libros y ensayos, entre los cuales podemos mencionar $L o$ stupore di una vita che si rinnova (Roma: Cantagalli, 2008), Emergenza educativa - Che fare? (Roma: Fede \& Cultura, 2008), Con Galileo oltre Galileo (Roma: Sugarco, 2009), Parole di Fede ai Giovani (Roma: Fede \& Cultura, 2010), Perchè la Chiesa ha racione (Roma: Lindau, 2010) y Fede e Cultura. Scritti scelti (Roma: Jaca Book, 2011).. 
Finalmente, se concluye señalando lo siguiente: (a) el trabajo realizado por el papa Juan Pablo II durante su pontificado ha sido trascendental para dejar en claro que el hombre encuentra su fundamento en Cristo - verdadera identidad humana-; (b) la Iglesia, gestora de la humanidad, recibe este legado para que a través de su Doctrina Social sea manifestado al hombre de hoy con el fin de que logre una vida más humana.

Palabras Clave: Doctrina Social de la Iglesia, modernidad, laicismo, Iglesia, nueva evangelización, humanidad, Cristo, Juan Pablo II.

\section{JOHN PAUL II'S CONTRIBUTION TO THE SOCIAL DOCTRINE OF THE CHURCH}

Abstract: This paper aims to highlight the contribution made by Pope John Paul II to strengthen the Social Doctrine of the Church. Firstly, the analysis of the legacy left by four of his predecessors, Pius IX, Leo XIII, Pius XI and Pius XII, who all had a major involvement in the life of the Church to face the onslaught of modernity, is made. Secondly, it focuses on John Paul II's figure for an analysis of his performance in the Church's life from the perspective of the «new evangelization». For this purpose, we make a review of the four documents in which John Paul II addresses critical aspects of human life such as Redemptor Hominis, Lavorem Excersens, Rei Socialis and Centesimus Solicitudo Annus. Finally, the paper is concluded by stating the following: (a) the work done by Pope John Paul II during his pontificate has been crucial to make clear that man finds his rationale behind Christ true human identity, (b) the Church, responsible of mankind management, receives this legacy in order to be manifested to today's man through the social doctrine so that he achieves a more human life.

KeYwords: Social Doctrine of the Church, modernity, laicism, Church, new evangelization, humanity, Christ, John Paul II. 


\section{INTRODUCCIÓN}

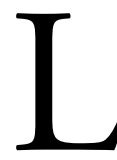

a Doctrina Social de la Iglesia nace en los últimos 150 años no para responder a problemas particulares, sino que nace del desencuentro entre la Iglesia y la modernidad. Recordemos que para la modernidad la política era todo. Se trata de un mundo construido sobre la razón sin Dios al servicio del poder.

El hombre moderno conoce científicamente y organiza los objetos, y el único objetivo de la razón humana es organizar las cosas para el hombre. La modernidad quiere un humanismo sin Dios, por eso divide ciencia y fe. Hay un ateísmo que se revela progresivamente en el tiempo. Pablo VI había percibido en profundidad la crisis moderna y la ha expresado en la carta Evangelii Nuntiandi.

La modernidad se juega el destino del hombre en la Política y en el Estado. A partir de la Revolución Francesa, el Estado se asume la función de hacer feliz al hombre; la Ilustración es el predominio de la inteligencia que impone la virtud política con violencia. La verdad entonces ya no es la revelación de lo que es, sino de lo que se construye solo con la razón.

Esta es la cuestión de la Doctrina Social de la Iglesia: oponer al ateísmo una doctrina libre y abierta, ya que se puede ser moderno sin ser ateos. Se trata de ofrecer otra visión de la vida, de una vida para la verdad y la libertad; esta es la presencia misionera de la Iglesia en la sociedad moderna. En la concepción de Estado se juega el destino del hombre. Este es el contexto de la Doctrina Social de la Iglesia.

Para tener una noción sintética se puede expresar en una contraposición de dos visiones de la realidad. A continuación se presentará esta idea: 


\begin{tabular}{|c|c|c|}
\hline Criterios & Principios laicistas & Principios cristianos \\
\hline Persona & $\begin{array}{l}\text { Prioridad sobre la } \\
\text { persona. } \\
\text { Primero la sociedad, las } \\
\text { leyes, las condiciones y } \\
\text { condicionamientos. } \\
\text { Si uno posee las leyes de la } \\
\text { sociedad hace un hombre } \\
\text { distinto, de acuerdo a ella. } \\
\text { La sociedad hace al } \\
\text { hombre sujeto de algunos } \\
\text { derechos. }\end{array}$ & $\begin{array}{l}\text { Prioridad de la persona sobre la } \\
\text { sociedad. } \\
\text { El hombre tiene conciencia de la } \\
\text { realidad, es un hombre que conoce y } \\
\text { ama a los hombres que encuentra. La } \\
\text { sociedad nace del encuentro entre el } \\
\text { hombre y la mujer; la familia es la célula } \\
\text { de la sociedad. El pueblo defiende su } \\
\text { tierra, se da su idioma, es la base de la } \\
\text { cultura. Este hombre tiene conciencia } \\
\text { de persona y de pueblo. A su vez se } \\
\text { da instituciones para defenderse. La } \\
\text { sociedad nace de abajo, es decir del } \\
\text { hombre que vive. }\end{array}$ \\
\hline Sociedad & $\begin{array}{l}\text { Prioridad del Estado } \\
\text { sobre la sociedad, } \\
\text { c o n s i d e r a d a } \\
\text { desordenada y violenta. } \\
\text { El Estado es el orden } \\
\text { (Hobbes), es la sociedad } \\
\text { pensada. } \\
\text { El hombre se vuelve lobo } \\
\text { para el hombre, por eso } \\
\text { se necesita un Estado } \\
\text { fuerte que aparece como } \\
\text { la sociedad perfecta. }\end{array}$ & $\begin{array}{l}\text { Prioridad de la sociedad sobre el } \\
\text { Estado. } \\
\text { El Estado es una ayuda para la sociedad. } \\
\text { Santo Tomás decía que el Estado debe } \\
\text { tender a servir al bien común de la } \\
\text { sociedad. Por tanto, el estado es legítimo si } \\
\text { busca el bien común. } \\
\text { León XIII explicó estas verdades en } \\
\text { las encíclicas Libertas, Inmortale Dei. } \\
\text { No se trataba de un Estado laico no } \\
\text { confesional. } \\
\text { El Estado está al servicio de la sociedad. } \\
\text { No interesa la forma de gobierno, lo que } \\
\text { interesa es que sirva al pueblo. }\end{array}$ \\
\hline
\end{tabular}




\begin{tabular}{|c|c|c|}
\hline $\begin{array}{c}\text { Relación } \\
\text { Iglesia y } \\
\text { Estado }\end{array}$ & $\begin{array}{l}\text { Separación de la } \\
\text { Iglesia del Estado. } \\
\text { Es un principio de la } \\
\text { primera Revolución } \\
\text { Francesa. } \\
\text { Esta separación se } \\
\text { vuelve sujeción de la } \\
\text { Iglesia al Estado. } \\
\text { La Iglesia si tiene una } \\
\text { expresión lo es bajo el } \\
\text { Estado. } \\
\text { De esta manera se crea } \\
\text { el concepto de Religión } \\
\text { del Estado, formulado } \\
\text { en la Iglesia Protestante. }\end{array}$ & $\begin{array}{l}\text { Distinción entre la Iglesia y el } \\
\text { Estado (con papa Gelasio). } \\
\text { La Iglesia es una cuestión de la } \\
\text { conciencia y de la vida de la Iglesia. } \\
\text { El Estado no tiene poder sobre la } \\
\text { Iglesia. } \\
\text { Los laicos tienen que intervenir en la } \\
\text { política. } \\
\text { Esta distinción ha salvado la } \\
\text { conciencia de los pueblos. }\end{array}$ \\
\hline
\end{tabular}

Emerge el concepto de libertad de la Iglesia como libertad para ejercer la misión. Los Concordatos han sido hechos para salvar la libertad de la misión de la Iglesia, condicionada por los regímenes políticos, no por su aspiración hegemónica. La Iglesia es una realidad presente dentro de la vida del pueblo como servicio.

\subsection{La herencia que Juan Pablo II recibe de la Iglesia}

Existen algunos puntos de enlace con el pasado que son determinantes. Estos son los que siguen:

- Pío IX: Un primer momento lo encontramos con el considerado Papa enemigo del laicismo. Este Sumo Pontífice tuvo una conciencia clara del desarrollo del laicismo y fijó las distancias entre el laicismo 
y el cristianismo. Había comprendido que la lejanía del hombre de Cristo es una distancia del hombre de sí mismo.

Un punto significativo fue manifestado en el Syllabus, es decir, en las 30 proposiciones laicistas condenadas por la Iglesia. El Papa pasa de los principios de orden filosófico a los principios de la relación entre fe y razón. El documento es clarísimo e iluminador acerca de la naturaleza del laicismo.

Toda la grandeza de la Iglesia en el siglo xx fue el defender su diferencia con el Estado. La grandeza de una sociedad depende de la variedad, de las diferencias. La Iglesia, al trabajar por la libertad de los cristianos, lo ha hecho por la libertad de todos los hombres. Somos distintos, debemos compararnos a partir de las diferencias, ya que esta da la posibilidad del diálogo.

En cambio, el objetivo de la sociedad laicista es que no existan diferencias. La Iglesia no podrá aprobar el Estado como origen y fuente de todos los derechos, ni un Estado que goza de un derecho sin límites. Tal Estado es el autoritarismo total, en el cual ya no existen límites para él.

Para la Iglesia el Estado tiene el límite objetivo de la persona, de la conciencia, de las relaciones parentales; estos son límites que el Estado tiene que reconocer. Hay derechos de la naturaleza humana que son previos y proceden del hecho fundamental de que el hombre es imagen y semejanza de Dios.

- León XIII coincide con el nacimiento formal de la Doctrina Social de la Iglesia. Para él la sociedad es el lugar de la libertad, una libertad que no es instintividad. Pero tenemos que acordar que sin 
verdad no hay libertad y esta no es la libertad de escoger, sino que es responsabilidad de la persona frente a la historia, frente a sí misma, frente a Dios. La sociedad se construye en el ámbito y respeto de la libertad. Por eso, la imagen del Estado debe ser la del servidor del bien común, de la totalidad de la persona y de la sociedad.

En Rerum Novarum (RN), León XIII afronta la situación de los trabajadores. Era el tiempo de la lucha entre dos sistemas: Liberalismo y Socialismo Colectivista. Sin embargo, la Iglesia no propone un tercer camino, sino que se juega con la caridad.

En primer lugar, la Iglesia defiende el derecho fundamental de la propiedad, aunque la propiedad no es presentada como un valor absoluto. Afronta el problema teniendo en cuenta todos los factores; configura una visión realista de la vida social, no desde la perspectiva de la ideología, sino desde una hipótesis de solución de una presencia social en la economía. El nuevo planteamiento consiste en vivir la libertad.

León XIII tuvo la conciencia del tiempo que le tocaba vivir. Es así que, propone la grandeza de la tradición cristiana.

- Pío XI vivió en su pontificado un tiempo caracterizado por el laicismo y los totalitarismos, entendidos como fuerza religiosa. El totalitarismo es intrínsecamente perverso porque destruye al hombre.

La labor de Pío XI fortalece la conciencia cristiana porque retoma la vida religiosa de la Iglesia como misión en el presente. En esta realidad comprendió que el pueblo y la familia son fundamentales; se trata de una organización de las fuerzas que intervienen en la 
sociedad. Fue el Papa de la Acción Católica, de las instituciones seculares. Fue el comienzo del pluralismo eclesial que se expresaría luego con amplitud en el Concilio Vaticano II.

Su encíclica Quadragesimo Anno (QA) realiza una lectura profética de los límites del estatismo, leyendo más allá de las apariencias. La exigencia fundamental era la que llamará cultura. La cultura es la conciencia crítica y sistemática de la realidad que se debe respetar y favorecer.

En la sociedad existen por un lado la familia que tiene derecho a educar y por otro los grupos sociales que crean realidades culturales. El Estado debe ayudar a realizar estos derechos, no puede sustituirse ni a la familia, ni a los grupos. Aparece ya delineado el Principio de Subsidiariedad del Estado que está en el origen de las instituciones. El Estado ayuda a la sociedad para que sea ella misma, no se impone sino que sirve; cuando el Estado realiza este servicio es él mismo, de lo contrario es una realidad negativa.

- Pío XII hace ver cómo el laicismo acabará en un desastre, ya que ese proyecto de una humanidad sin Dios terminará por destruir al hombre. Un hecho patente de esto se manifiesta en la Segunda Guerra Mundial.

El Papa comunica una certeza de fondo: si la fe vuelve a ser vivida puede nacer un tiempo de libertad y de paz. Por eso reclama a la Iglesia su actividad misionera. Advierte el peligro de la masificación y alude a la ideología del «modo americano de vivir».

Hay un cambio fundamental en la Doctrina de la Iglesia frente a la dictadura del siglo XXI, que es la dictadura del tecnicismo 
que dispone del hombre. El magisterio de Juan Pablo II planteó este álgido problema en la encíclica Evangelium Vitae (EV).

\subsection{La herencia Que entrega Juan Pablo II}

Juan Pablo II comienza recibiendo el magisterio del Concilio Vaticano II y del clarividente Pablo VI. Sintetiza su tarea en una frase: la «nueva evangelización», que vibra ya desde su primera encíclica Redemptor Hominis $(\mathrm{RH})$. En ella, vuelve a proponerle Cristo al hombre como acontecimiento de vida; define la misma evangelización como reabrir el diálogo entre Cristo y el corazón humano. La Iglesia es misionera porque sirve a este diálogo viviente, y este es posible porque el corazón del hombre está vivo. El Papa vio que la modernidad termina con la muerte del hombre; para el ateísmo el hombre le pertenece al mismo hombre. El ateísmo no es un fenómeno de hoy, sino que hoy se teoriza sistemáticamente y se lleva a la práctica.

La originalidad de Juan Pablo II fue descubrir que el hombre resiste frente a los repetidos embates del ateísmo. La invasión de las doctrinas y prácticas hacen surgir en corazón del ateísmo un innegable despertar religioso; por eso el hombre resiste al ateísmo.

La genialidad de Juan Pablo II es que ha sabido entrar en la profundidad del corazón del hombre de hoy y ha descubierto su deseo de vivir. Es por eso que desde el primer día de su pontificado dijo: «Abran las puertas a Cristo».

Desde entonces la Iglesia ya no se plantea el problema de las ideologías, sino la tarea de revelar al hombre la verdad del hombre que se manifiesta en el Hombre por excelencia. La humanidad de Cristo se ha convertido en el lugar en donde el hombre puede encontrar su humanidad. 
Esta es la forma como Juan Pablo II toma la herencia del pasado volviéndose al corazón del hombre.

El Papa manifiesta que su certeza es la de la Iglesia, expresada muy bien por Pascal: El hombre supera al hombre, es más que todo el mal que hizo y padece; es un infinito indestructible. ${ }^{1}$ Los totalitarismos han acabado con el físico de millones de personas, con la moral y la cultura de muchos hombres; de hecho los antropólogos hablan de un cambio antropológico. Pero el corazón del hombre es invencible porque ha sido salvado por la muerte y resurrección de Jesucristo (RH 13-14).

Si la Iglesia debe volver a abrir el diálogo entre Cristo y el hombre, la Doctrina Social hace concreto e histórico este diálogo. De manera que muestra al hombre el verdadero Hombre y le señala que este tipo de hombre es quien puede crear un mundo más humano. Esta realidad provoca plantear dos preguntas esenciales: (a) ¿en qué sentido Cristo revela al hombre su identidad?² y (b) ¿`cómo el Hombre verdadero cambia la historia?

\section{MAGISTERIO DE JUAN PABLO II}

Para el análisis y desarrollo de las cuestiones planteadas se hace necesario realizar una revisión del corpus de los documentos propuestos por Juan Pablo II durante su pontificado. En ellos, Juan Pablo II procura mostrar al hombre la clave para afrontar las consecuencias de las ideologías de la modernidad. Los documentos citados a continuación tienen como primordial función

1 Véase para mayor información el portal del Vaticano. Disponible en <http://www.vatican. va/holy_father/benedict_xvi/audiences/2012/documents/hf_ben-xvi_aud_20121107_ sp.html>. Consulta hecha en 20/01/2014.

2 Nos puede dar la pista la frase de Pilatos: "Aquí tienen al hombre» (Jn 19,5), aunque Pilatos no lo sabe, lo señala: aquí está la humanidad verdadera, esa humanidad que da la verdadera estatura del ser humano. 
develar al hombre la «verdad del hombre», es decir Cristo, verdadera humanidad e identidad del hombre.

-Redemptor Hominis (RH), 1979: 'El Redentor del hombre', primera encíclica escrita por Juan Pablo II, analiza los problemas contemporáneos del hombre y traza las pautas para una respuesta a partir de la verdadera comprensión del ser humano.

-Evangelium Vitae (EV), 1995: 'El evangelio de la vida', en esta encíclica el Papa se preocupa por evidenciar y rescatar el valor y el carácter de inviolable de la vida de todo hombre.

-Laborem Excercens (LE), 1981: Esta encíclica se publicó en el 90. ${ }^{\circ}$ aniversario de la Rerum Novarum y aborda la concepción del hombre desde la perspectiva del trabajo como una actividad que dignifica la realización humana.

- Sollicitudo Rei Socialis (SRS), 1987: Esta encíclica se escribió con ocasión de los 20 años de la Populorum Progressum, manifiesta la preocupación social de Iglesia y aborda los temas fundamentales de la problemática del hombre como desigualdad social y económica, destino universal de los bienes y las ventajas y peligros del desarrollo. - Centesimus Annus (CA), 1991: Juan Pablo II, con ocasión de los 100 años de la Rerum Novarum, escribe esta encíclica con el propósito de actualizar el trabajo de sus predecesores en relación a los problemas actuales del hombre y del desarrollo de los pueblos.

\section{LECTURA DE LA MODERNIDAD POR EL PAPA JUAN PABLO II}

En la encíclica Redemptor Hominis (RH) se encontró una pista orientadora acerca de las cuestiones planteadas en este trabajo. Se nos dice que el hombre 
es el camino de la Iglesia hacia la verdad, que se opera en el encuentro con Cristo. Si ambos caminan juntos el hombre y Cristo, es posible encontrar la verdad que aspira el ser humano (cf. RH 15-17).

La Iglesia, que camina abriéndose al hombre, ¿cómo percibe a la modernidad? El Papa observa que en la modernidad aparecen dos palabras inquietantes:

a) La humanidad está alienada por el «miedo». Justamente la ausencia de miedo es signo de la verdad. Pero ¿̇de qué tiene miedo el hombre de hoy? Es que, junto con los medios que el hombre tiene a disposición, este percibe una amenaza, ya que el mismo hombre puede utilizarlos en contra del hombre. Esto pone en evidencia que no es ni la ciencia ni la técnica lo que le da seguridad a la humanidad; lo que le da seguridad es la madurez del hombre. Vemos que estos medios dan poder mas no madurez.

La humanidad exige ser más y no tener más. El progreso técnico no es suficiente para asegurar la plena humanidad. El instrumental que el hombre tiene es más bien una amenaza de violencia, porque frecuentemente lo usa para dominar. El problema no está en el poder, sino en la verdad.

Es por eso que al fin de la modernidad el hombre aparece pequeño, no tiene criterio ni sensatez para usar el poder. El hombre culto es el que tiene conciencia de su identidad, es quien puede usar de las cosas. En realidad este miedo abre la pregunta: ¿estamos progresando o retrocediendo?

Se constata que el hombre no es capaz de realizarse solo con los medios de la ciencia y de la técnica; necesita vivir el problema 
de la verdad. Debe aprender a desvelar el Misterio para encontrar el sentido de la vida que ha perdido.

Juan Pablo II ha enseñado, no solo a los cristianos, sino a todos los hombres, que se puede ir más allá del poder, que se puede llegar a la verdad y entonces abre la pregunta sobre Dios. No sabemos cuál es el rostro de la posmodernidad, pero el hombre debe saber que puede no morir si reabre la cuestión de su identidad; y solo Cristo puede darle la respuesta (cf. RH 16).

b) La segunda palabra que aparece en el balance de la modernidad es la «mentira». El hombre que no vive en la realidad vive en la mentira.

El Papa enseńa genialmente en qué consiste la mentira. Al hombre delineado por la política se le dice que es libre, pero en realidad se le obliga a obedecer, se le considera un número; los derechos humanos proclamados son vacíos y son escasas las situaciones en que han sido experimentados. La política no crea el bien; es positiva si el hombre la vive buscando el bien, de lo contrario es mentira (cf. RH 17).

El siglo xx se ha vivido en esta mentira, pero la puede superar retomando la cuestión de la verdad, y Dios que es la verdad sigue hablando al hombre de hoy. Es así como Juan Pablo II ha penetrado con el hombre en la modernidad, hablando no solo a los cristianos, sino a todos los hombres que buscan la verdad.

Más allá de los límites de la modernidad está presente la búsqueda de lo bueno, de lo bello, de lo verdadero. La Iglesia recorre este camino y llama al hombre a recorrerlo. La Doctrina Social de la Iglesia enseña cómo el hombre puede cambiar la historia. Asimismo, 
Cristo revela al hombre toda la verdad del hombre. Naturalmente, el hombre busca esta verdad que Dios le ofrece; es responsabilidad suya seguir buscándola (RH 10).

\subsection{RECURSOS QUE EVIDENCIAN LA REALIZACIÓN DEL HOMBRE}

\section{El primer recurso social es la cultura}

El hombre es sujeto de cultura, es el modo con que vive su humanidad; vive en tensión para descubrir el sentido de su vida. La cultura se refiere al ser, no al tener; en esto consiste la impostación del problema humano. La cultura no es suma de cosas, sino la verdad que madura en el corazón del hombre. En nuestra cultura, el hombre es pobre respecto al ser aunque sea más rico en el tener. El hombre moderno es un bárbaro, es decir, no tiene el sentido de la realidad y de la historia. El tener pertenece a la cultura secundaria y no es sobre esta donde se juega el destino del hombre, sino sobre la cultura primaria del ser y de su verdad.

La cultura nace de la libertad. El corazón del hombre no puede sufrir condicionamientos totales por mucho tiempo, prueba de ello es cómo su ansia de libertad sobrevivió a los regímenes totalitarios. Por lo tanto, el primer gran recurso social del hombre es la cultura que es un movimiento de humanización. Mientras la cultura humaniza, la ideología deshumaniza. El hombre que supera la modernidad puede hacer cultura, tomando conciencia de su humanidad. Lo importante es mantener abierta la pregunta por el sentido de la vida. Si el hombre no se pregunta por el sentido de la vida es inútil decirle que Cristo es el sentido de la vida.

La Doctrina Social de la Iglesia se propone hacer cultura con su razón. Hoy, el problema de la vida no es saber si Dios existe o no, sino saber 
cómo ser felices. Pero el hombre sin Dios no es feliz y se desintegra. Es necesario plantear el problema de la vida, es decir, de la cultura. El primer recurso social es ayudar a vivir. Este recurso, evidentemente es teórico, ya que trata de conocer la realidad, pero el conocimiento señala la verdad.

\section{El segundo recurso social es el trabajo}

Este tema se trata en Laborem Excercens (LE). Es el hombre quien ejerce el trabajo y quien vive esta dimensión de la vida. En el trabajo el hombre juega su cultura en la realidad, haciéndola coherente con la visión que tiene de la realidad. La cultura debe terminar en el trabajo según la verdad.

La verdadera diferencia entre el hombre y el animal no está en la razón, sino en el trabajo. Hoy tenemos diferentes tipos de inteligencia artificial, pero ningún instrumento trabaja, ninguna inteligencia artificial puede transformar la realidad. Un trabajo sin cultura es un pragmatismo que no deja huella en la historia. El hombre entra en la sociedad con dos instrumentos: la cultura y el trabajo, y con ellos llega a la plenitud de su humanidad; se trata de una circularidad que no termina y el hombre es quien realiza este pasaje.

Por tanto, el trabajo no son las coyunturas económicas, políticas o históricas. El trabajo tiene aspectos objetivos, que se pueden reducir a leyes (trabajo tecnológico), pero la tentación es pensar que el trabajo es cuestión de tecnología. Hay una dimensión subjetiva del trabajo que no puede anularse por la dimensión objetiva. El sentido subjetivo del trabajo está dado por el hecho de que quien realiza el trabajo es un hombre y en ello expresa su humanidad, dominando la realidad. Juan Pablo II habla del «Evangelio del trabajo», que proviene de toda la tradición cristiana. Entonces lo que 
determina el valor del trabajo es que es el hecho de que sea realizado por una persona; en esto consiste su dignidad.

El fin de cualquier trabajo es la realización del hombre. A través de la acción, el hombre realiza lo verdadero, y su importancia consiste en que en esta tarea el hombre se juega todo su ser. No se puede alterar la primacía de la subjetividad sobre la objetividad del trabajo. El problema del trabajo está en la consideración de quién es el que trabaja y cómo lo realiza. Por ello, los protagonistas del mundo del trabajo son los hombres del trabajo, no los trabajadores enunciados en un rol; es en el trabajo donde el hombre expresa su humanidad.

Este aspecto fundamental del trabajo se enriquece con el valor de la solidaridad operativa. Los hombres del trabajo enfrentan la vida social jugando en el trabajo su humanidad. El trabajo no es una particularidad tecnológica o económica (cf. LE 6). En la encíclica, el trabajo aparece como un bien del hombre como tal. El problema del trabajo no es el choque entre capital y trabajo. Lo decisivo es que el trabajo sea considerado en su pertenencia al hombre y a su dignidad (cf. LE 9-10).

La familia y la nación nacen del trabajo de la persona, y por eso es el hombre quien crea la sociedad. Ciertamente no es solo creación de la persona, hay pesados condicionamientos ideológicos, pero no sustituyen el planteo del trabajo.

Si se contrapone el capital y el hombre, uno de los dos debe ser suprimido; se debe superar esta imagen ideológica del trabajo, afirmando su verdadera dimensión (cf. LE 13). El economismo como el materialismo son dos planteamientos equivocados. El centro está en la personalidad que trabaja, aquí está el punto de vista original para enfrentar todos los problemas del trabajo. Se trata de poner todos los problemas del trabajo en 
los derechos y dignidad del hombre que trabaja. El trabajo es una realidad móvil porque los hombres que trabajan son dinámicos.

En LE, vemos que los sindicatos no están planteados como estructuras de reivindicación, sino con una función educadora para formar en la moral del trabajo. Al final de la encíclica, el Papa individualiza la responsabilidad de la Iglesia, que es de carácter educativo, teórico y práctico. La cultura humana y el trabajo expresan al máximo la fe y la dimensión ética del hombre. La Iglesia enseña que el trabajo es un valor fundamental en la Revelación cristiana.

El valor del trabajo se desarrolla en la «espiritualidad del trabajo». El trabajo es la participación a la obra creadora de Dios, por eso todo trabajo eleva al hombre sobre sí mismo; en él, el hombre respira una dimensión de infinito. Se trata de una experiencia análoga a la del amor entre el hombre y la mujer; en el amor humano se respira algo de infinito. En el trabajo y en el amor el hombre colabora con Dios.

Estas son las condiciones para afrontar el trabajo. Cristo es el hombre del trabajo, no solo porque trabajó como hijo del carpintero, sino porque para nosotros el hijo del carpintero es el Hijo de Dios que trabaja. Por lo tanto, el cepillar, clavar, encolar es trabajo del Hijo de Dios. Pero el trabajo del Hijo ha alcanzado su culmen en el trabajo de la Pascua.

No podemos pretender que el trabajo no sea sacrificio, ya que hace vivir de alguna manera la muerte, pero también la resurrección. Si junto a los hombres del trabajo están los hombres de fe, el trabajo se vuelve más vivo, se vuelve servicio al bien histórico de la humanidad.

3.2 Juan Pablo II, la DSI y el corazón del hombre actual 
Hay otra idea importante en la Doctrina Social de la Iglesia y es la demostración del realismo en el planteamiento de los problemas del sujeto; es al hombre en su identidad natural como cristiano, con su auténtica humanidad a la que debe dar una respuesta. La Doctrina Social trata de tener presente todos los factores de la realidad humana, tendrá que considerarlo como ser corporal y espiritual, individual y social. No sucede así con la ideología, ya que elimina todo lo que no concuerda con lo que propone como única verdad.

En la encíclica Sollicitudo Rei Socialis, Juan Pablo II presenta dos nudos importantes de su Doctrina Social. Es publicada para conmemorar los 20 años de Populorum Progressio; que aborda el tema del desarrollo de los pueblos. En ella, Pablo VI advirtió que el progreso corría el riesgo del tecnicismo. Podemos decir que el tema del desarrollo era más amplio y realista que el de la globalización.

Plantea el desarrollo en perspectiva mundial, por tanto los problemas debían ser tratados a nivel universal. No podía verse el progreso $\precsim$ de una parte del mundo contra la otra. Asimismo, advierte con claridad que el desarrollo es el nuevo nombre de la paz, en la que cada pueblo de la tierra tiene derecho al desarrollo.

El Papa señala que una dificultad para el progreso es la diferencia entre el Norte y el Sur del mundo. No se trata de áreas geoeconómicas o geopolíticas, sino que se trata de dos modos de vivir. El desarrollo tiene que promover una vida digna en la que todos los pueblos tengan derecho a la iniciativa económica, dando su contribución específica (cf. SRS 14).

No existe una economía mundial en sentido unívoco, ya que se dan una variedad de contribuciones y el mercado mundial se crea a partir de esa pluralidad de iniciativas. La objeción para el compromiso pluralista 
en el desarrollo nace de la antigua tentación de los totalitarismos europeos. El freno del desarrollo es la intervención del Estado que, si no cumple adecuadamente su rol, detiene iniciativas populares.

Ningún grupo social o partido puede usurpar el rol de único guía, porque ello implica la destrucción de la subjetividad de la sociedad, de todas las presencias sociales. El desarrollo está amenazado por un nuevo totalitarismo. Frecuentemente, se puede observar que países en vías de desarrollo se convierten en piezas de un mecanismo gigantesco que destruye la individualidad. Cada parte debe asumir su propia responsabilidad. El desarrollo es la energía de la cultura y del trabajo, de lo contrario se cae en otra esclavitud.

La Doctrina Social nos exige partir de la base de la sociedad: del sujeto, de la familia, de los pueblos que poseen sus propios dinamismos de desarrollo. La unidad impuesta siempre es nefasta. El desarrollo se manifiesta como solicitud por la paz. Para un auténtico desarrollo humano es indispensable la posibilidad de vivir libremente la cultura y el trabajo:

a) Es necesario superar la contraposición entre el ser y el tener; no hay desarrollo sin cultura.

b) Es importante el protagonismo de la libertad personal, que no puede subordinarse a un ideal abstracto de desarrollo.

Cultura y libertad se iluminan con la Palabra Revelada. El desarrollo no es un ideal acabado acerca de la vida y de la historia, sino que es la expresión histórica de la vida realizada en Jesucristo en el hoy de cada circunstancia concreta. El desarrollo es una modalidad histórica de testimoniar la resurrección de Cristo. 
La Iglesia con su acción impide la rigidez ideológica; no hay nada absoluto, lo único absoluto es Cristo resucitado que vive en el hombre concreto. La fe se expresa en forma de cultura. Esta experiencia vuelve apasionante la obra de la historia, impidiendo la idolatría. La Doctrina Social de la Iglesia realiza una nueva evangelización contribuyendo al verdadero desarrollo de la humanidad. Las diferentes situaciones de cada pueblo no deben ser un obstáculo.

Los cristianos colaboran en la empresa del desarrollo sin recetas, se ponen a transitar por el camino de la comunidad humana en una búsqueda constante de las sendas más acordes a la naturaleza del hombre; los totalitarismos proponen recetas. La vida social es un campo de colaboración, no un lugar de hegemonía de una determinada ideología.

La virtud típicamente social es la solidaridad. Esta es la expresión laica de la caridad. Ella hace sensibles a las vicisitudes de los hombres y percibe como esencial el trabajar "con»; esta solidaridad se verifica en el colaborar en el bien con quien sea. Se trata de la tarea común de la creación de una situación social que exprese la variedad de la realidad (SRS 31).

La Iglesia exige la libertad de estar presente en este mundo, la libertad de proponer a Cristo al hombre de hoy, viviendo la solidaridad con todos los hombres. No tenemos una idea general que viene de lo alto. La ciudad de Dios se va formando con la levadura de nuestra vida, en el esfuerzo de que la vida terrena sea más humana, donde la Iglesia está presente libremente, la sociedad se va haciendo más humana.

El valor de esta encíclica es haber hecho comprender la fascinación de esta obra. Se actualiza en ella el lema de san Benito Ora et lavora.

En su encíclica Centesimus Annus (CA), el Papa tiene presente el año 1989 en el que se marca el fin de las grandes ideologías totalitarias, el fin 
del marxismo. Esta lectura de los acontecimientos constituye una novedad en la doctrina de Juan Pablo II: revive la doctrina de la $\mathrm{RH}$ reformulando la Doctrina Social de la Iglesia. Pero el Papa plantea un cambio de rumbo en continuidad con la Doctrina Social; realiza una nueva lectura del presente para proyectarse hacia el futuro. Con esto nos recuerda que una fe que no lee los signos de los tiempos está muerta o se mueve en la ideología (CA 3).

De la RN queda la grandeza del choque entre el capital y el trabajo, con sus diversas formas y el capitalismo como una presión mundial frecuentemente difícil identificar. La solución del choque pertenece a la vida de los hombres del trabajo.

Aquí Juan Pablo II repropone el derecho a la propiedad, derecho fundamental de la persona. León XIII había enseñado que hablar de estos problemas no es estar ajeno a la acción pastoral de la Iglesia; no hay auténtica pastoral si no se afronta la vida de los hombres. La Doctrina Social se presenta como capacidad de juicio y acción sobre la vida en toda su realidad. Los totalitarismos tratarán de reducir la obra de la Iglesia a la sacristía, al intimismo, a una vida espiritual desencajada de la vida diaria. El laicismo quería reservarse la sociedad como lugar de acción.

En la tercera parte de la CA, el Papa parte del año 1989, pero afirma que, de hecho lo acontecido ya había ocurrido en 1945 con la tragedia de la Segunda Guerra Mundial. El 1945 fue el año de una gran mentira, el comunismo soviético pretendió haber ganado la guerra manteniendo viva su ideología; solo el Magisterio de la Iglesia había denunciado estas cosas. El marxismo no fue combatido por la Iglesia por sus ideas de justicia social, sino por su totalitarismo.

Luego encontramos una nota humilde, pero importantísima. Juan Pablo II expone en esta encíclica su visión personal, como hipótesis de 
trabajo para entender este tiempo; es decir, no está haciendo Doctrina de la Iglesia, sino que parte de la encíclica queda como una lectura abierta para la investigación.

Lo que caracteriza al 1989 es la caída de regímenes opresores sin derramamiento de sangre; en este periodo se evidenció una revolución sin violencia, contraria a las ideologías del 1800 . Fue la gran revolución de la época en la que no fue necesario matar al opresor, porque se realizó a través de la razón.

El segundo factor de la caída de estos regímenes fue la conciencia personal de los laicos cristianos que se enfrentaron a la ideología, en casi todos los países de Europa, al concluir la Segunda Guerra Mundial. Estos trataron de construir un mundo diverso basado en el respeto a la dignidad humana. Nuevamente el año 1989 es el testimonio de la fuerza del pueblo que piensa y que asume la responsabilidad de su vida llevando la novedad de la vida de Cristo.

El tercer factor de la caída de dichos regímenes es la crisis de Э la economía debida al irrealismo de la ideología. Esta había modificado economías ya consolidadas, como en el caso de Polonia, que contaba con una economía agrícola rentable y fue arbitrariamente sustituida por una pesada industrialización que terminó por hundir la agricultura.

Encontramos otra afirmación capital: la verdadera causa de la caída de estos regímenes es el vacío espiritual del hombre ateo. El ateísmo es un vacío de valores vivido como una tragedia ya que no promete vida plena (CA 24). La novedad en la encíclica es la certeza de Juan Pablo II de que es posible un encuentro entre Cristo y el corazón del hombre.

Pero la historia de los países liberados del comunismo es la demostración de que no se pasa mecánicamente del vacío de la vida a la 
novedad; se necesitaba la obra de la educación en todo el mundo, no solo en el Este europeo. El peso de la injusticia de la violencia de los totalitarismos no cae mecánicamente, se necesita de un tiempo para realizar un camino de formación de la conciencia, de este modo puede ir surgiendo un hombre nuevo.

En la cuarta parte de la encíclica se invita a redescubrir el destino universal de los bienes. El problema económico hay que retomarlo nuevamente. La nueva economía debemos crearla juntos.

Plantea también el problema de la ecología, que en términos reales es un problema de educación que comienza desde la familia. Todos debemos contribuir a la nueva economía con la cultura y el trabajo.

En la quinta parte se plantea el problema del futuro y es la cultura la gran empresa de la vida. Emerge la responsabilidad de la Iglesia que es antitotalitaria (CA 45). La presencia de la Iglesia proclama los criterios de discernimiento sobre el bien y el mal; ella los busca en la ley natural que Dios ha puesto en cada hombre y en la Revelación. Esta es una cuestión fundamental para la vida humana.

Es necesario entender que para la construcción del mundo hay que recuperar la importancia de la cultura. Asimismo, es preciso vigilar contra el totalitarismo del Estado, reformulando la verdadera conciencia del pueblo.

\section{CONCLUSIONES}

La Doctrina Social de la Iglesia es necesaria para expresar la misión de la Iglesia en el hoy de la historia. Actualmente, tenemos el peligro de la tecnociencia que pretende ser el valor absoluto; la Doctrina Social está en este mundo para que el hombre logre vivir más humanamente, en esta 
situación, solicitando la autenticidad humana que es fruto de una vida de fe y de una seria búsqueda de la razón.

La Iglesia tiene la capacidad y la misión de cambiar la historia con la centralidad del hombre en su relación con el Misterio. Es decir, con el Hijo de Dios llamado a participar de nuestra misma naturaleza en la construcción de esta historia.

Por otro lado, la Doctrina Social es el empuje para la actividad de los hombres. Está destinada a demostrar que la fe humaniza la vida, expresa la responsabilidad del presente y del futuro, y se coloca en el umbral del nuevo siglo. La novedad de las cosas deriva de la infinita potencia de Dios, capaz de hacer nuevas todas las cosas. La nueva creación está dentro del corazón del bautizado, que participando de la vida de la Iglesia abraza a todos los hombres (CA 62).

La vida, animada por la razón y el trabajo, está abierta a la fe y esta es una realidad maravillosa. Desde hace 2000 años la Iglesia anuncia al hombre: «Tú puedes no morir», y lo hace mediante su Doctrina Social y en las obras. De esta manera va haciendo suyos todos los gozos, las angustias y las esperanzas de los hombres de nuestro tiempo.

\section{1 ResisTENCIA AL ATEÍ́SMO Y DESEO DE VIVIR}

Juan Pablo II descubrió que el hombre resiste frente al ateísmo. El despertar religioso surge en el corazón del ateísmo debido a la invasión de las doctrinas y prácticas. La genialidad de Juan Pablo II consistió en su capacidad para entrar en la profundidad del corazón del hombre actual y descubrir su deseo de vivir. Es por eso que desde el primer día de su pontificado dijo: «Abran las puertas a Cristo». 
A partir de ello, la Iglesia ya no se plantea el problema de las ideologías, sino la tarea de revelar al hombre la verdad del hombre que se manifiesta en el Hombre por excelencia. Es la humanidad de Cristo el lugar en donde el hombre puede encontrar su humanidad. Esta es la forma como Juan Pablo II tomó la herencia del pasado volviéndose al corazón del hombre.

Juan Pablo II manifestó que su certeza es la de la Iglesia. Los totalitarismos han acabado con el físico de millones de personas, con la moral y la cultura de muchos hombres; de hecho los antropólogos hablan de un cambio antropológico. Sin embargo, el corazón del hombre es invencible, porque ha sido salvado por la muerte y resurrección de Jesucristo. 


\section{BIBLIOGRAFÍA}

JUAN PABLO II

1979

1981

1987

1991

1995
Redemptor Hominis. Disponible en página del Vaticano <http://www.vatican.va/holy_father/ john_paul_ii/encyclicals/documents/hf_jp-ii_ enc_04031979_redemptor-hominis_sp.html>. Consulta hecha en 25/01/2014.

Lavorem Excercens. Disponible en página del Vaticano <http://www.vatican.va/holy_father/ john_paul_ii/encyclicals/documents/hf_jpii_enc_14091981_laborem-exercens_sp.html>. Consulta hecha en 25/01/2014.

Sollicitudo Rei Socilalis. Disponible en página del Vaticano <http://www.vatican.va/holy_father/ john_paul_ii/encyclicals/documents/hf_jp-ii_ enc_30121987_sollicitudo-rei-socialis_sp.html>. Consulta hecha en 25/01/2014.

Centesimus Annus. Disponible en página del Vaticano <http://www.vatican.va/holy_father/ john_paul_ii/encyclicals/documents/hf_jpii_enc_01051991_centesimus-annus_sp.html>. Consulta hecha en 25/01/2014.

Evangelium Vitae. Disponible en página del Vaticano <http://www.vatican.va/holy_father/ 
john_paul_ii/encyclicals/documents/hf_jpii_enc_25031995_evangelium-vitae_sp.html>. Consulta hecha en 25/01/2014.

\section{LEÓN XIII}

1891

Rerum Novarum. Disponible en la página del Vaticano <http://www.vatican.va/holy_father/ leo_xiii/encyclicals/documents/hf_l-xiii_ enc_15051891_rerum-novarum_sp.html>. Consulta hecha en 25/01/2014.

PABLO VI

1975

Evangelii Nuntiandi. Disponible en la página del Vaticano <http://www.vatican.va/holy_father/ paul_vi/apost_exhortations/documents/hf_p-vi_ exh_19751208_evangelii-nuntiandi_sp.html>. Consulta hecha en 25/01/2014.

PÍO XI

1931

Quadragesimo Anno. Disponible en la página del Vaticano <http://www.vatican.va/holy_ father/pius_xi/encyclicals/documents/hf_p-xi_ enc_19310515_quadragesimo-anno_sp.html>. Consulta hecha en 25/01/2014. 